\title{
Recurrent Pregnancy Loss in Women with Hashimoto's Thyroiditis with Concurrent Non-endocrine Autoimmune Disorders
}

\author{
Miriam Cellini, ${ }^{1, *, \dagger}$ Maria Giulia Santaguida, ${ }^{2, *}$ Ilaria Stramazzo, ${ }^{1}$ Silvia Capriello, ${ }^{1}$ Nunzia Brusca, \\ Alessandro Antonelli, ${ }^{3}$ Poupak Fallahi, ${ }^{4}$ Gargano Lucilla, ${ }^{2}$ Marco Centanni, ${ }^{1,2}$ and Camilla Virili ${ }^{1}$
}

Background: An increased rate of recurrent miscarriage has been described in patients with autoimmune thyroid disease. However, there is a lack of studies that assess the rate of recurrent pregnancy loss (RPL) in patients with Hashimoto's thyroiditis (HT) isolated or with concurrent non-endocrine autoimmune disorders (NEAD). The objective of the study was to assess the rate of RPL in patients with HT isolated or accompanied with non-endocrine autoimmune diseases.

Methods: This is a retrospective observational cohort study with a systematic review of the NEAD with concurrent HT in an outpatient Endocrinology Unit at a University Hospital. Among the 3480 consecutively examined women with HT, 87 patients met the criteria of RPL and represented the study group. Sixty-five of them had isolated HT and 22 women had HT+NEAD.

Results: The rate of RPL in women with HT was $2.1 \%$ versus $5.64 \%$ observed in women with HT+NEAD (odds ratio $=2.78[95 \%$ confidence interval 1.70-4.57]; $p<0.0001)$. On subdivision, this difference was still evident in euthyroid patients $(p<0.0001)$, while it disappeared in hypothyroid women $(p=0.21)$. The RPL did not correlate with the autoantibody concentrations nor in women with isolated HT nor in those with HT+NEAD. The presence of antiphospholipid syndrome (APS) explained RPL in 3 out of 22 (14\%) patients with $\mathrm{HT}+\mathrm{NEAD}$, the remaining being related to different autoimmune disorders. Interestingly, even subtracting the patients with APS, RPL was more frequent in patients with poly-autoimmunity than in patients with isolated HT $(p=0.0013)$.

Conclusions: The co-presence of NEAD is correlated with a higher risk of RPL in women with HT. The association with APS may explain only a fraction of RPL rate in patients with poly-autoimmunity.

Keywords: recurrent pregnancy loss, miscarriage, Hashimoto's thyroiditis, hypothyroidism, polyautoimmunity

\section{Introduction}

${ }^{\text {AU } 3>} \mathbf{M}$ ISCARRIAGE IS A spontaneous loss of the conceptus that occurs before 20 weeks of gestation (1). About $30 \%$ of all pregnancies result in miscarriage, and only one tenth of all miscarriages are clinically apparent $(2,3)$. Recurrent pregnancy loss (RPL) affects from $1 \%$ to $5 \%$ of couples and is defined as three or more consecutive recurrent miscarriages or, for many clinicians, as two or more consecutive miscarriages $(4,5)$. The etiology of recurrent miscarriage is multifactorial and remains unknown in $\sim 50 \%$ of women, despite detailed evaluation (6). In the remaining 50\% of the cases, the causes of RPL is explained by genetic, structural, infective, endocrine, thrombophilic, and immune factors (6). Autoimmune abnormalities play a relevant role in RPL and represent one of the several accepted causes of miscarriage (7). Among them, an increasing amount of evidence links thyroid autoimmunity with adverse pregnancy outcomes, including miscarriage or preterm deliveries. Indeed, Hashimoto's thyroiditis (HT), the most common autoimmune disorder worldwide (8), affects about $4 \%$ of women during their reproductive age $(9,10)$ and has been involved in the

AU2 ${ }^{1}$ Department of Medico-Surgical Sciences and Biotechnologies, "Sapienza" University of Rome, Latina, Italy.

${ }^{2}$ Endocrinology Unit, Santa Maria Goretti Hospital, AUSL Latina, Latina, Italy.

${ }^{3}$ Department of Clinical and Experimental Medicine, University of Pisa, Pisa, Italy.

${ }^{4}$ Department of Translational Research and New Technologies in Medicine and Surgery, University of Pisa, Pisa, Italy.

*These authors contributed equally to this article.

${ }^{\dagger}$ Present address: Endocrine, Diabetes and Andrology Unit, Humanitas Clinical and Research Center, IRCCS, Rozzano, Italy. 
pathogenesis of recurrent miscarriages (6). Noticeably, it may occur isolated or concurrent with other endocrine and/or non-endocrine autoimmune diseases, including the antipho-

AU4 spholipid syndrome (APS) (11), which may represent the clinical expression of a more extended derangement of the immune system. The presence of anti-thyroid peroxidase antibodies (anti-TPO-Abs) (about 10\% of women at 14 weeks of gestation) has been suggested as a cause of infertility and increased rate of miscarriage $(3,6,12-14)$. A metaanalysis has shown an odds ratio (OR) of 2.1 for RPL in women carrying anti-TPO-Abs (15), which were initially claimed as causative for single or repeated miscarriages (14). Nowadays, an appropriate balance between immunological factors of mother and fetus seems to be key in preventing an immune rejection of the embryo (16). In fact, a disruption of immunological self-tolerance and activation of a proinflammatory response has been linked to RPL $(17,18)$. However, no studies have examined whether the presence of more than one autoimmune disorder may increase or decrease the risk of miscarriages. Therefore, this study was aimed at evaluating the rate of RPL in patients with HT, isolated or concurrent with other non-endocrine autoimmune disorders (NEAD).

\section{Patients and Methods}

\section{Patients and study design}

This study has been carried out in a cohort of patients sequentially examined (2010-2016) and referred to our center for thyroid diseases in the outpatient Endocrinology unit. We examined the records of 9661 women with thyroid diseases and, among them, 3480 women had HT. In 3090 women, the HT occurred as isolated disease, whereas in 390 it occurred in association with other NEAD (i.e., vitiligo, chronic atrophic gastritis, coeliac disease, APS, etc.) (19). According to the policy of our center, during the initial interview, all women declared how many pregnancies and/or pregnancy losses they had during their reproductive life. For each patient, we recorded the number of these events, their age at the time of events, and, in case of miscarriage, the weeks of gestation when it occurred as well as the thyroid function, autoantibodies status, and sonographic pattern at the time of the first miscarriage. The diagnosis of HT has been confirmed in all patients in our outpatient section at the time of first examination but had been already done at the time of the first miscarriage. In the patients showing signs or symptoms and/or suspicion of additional autoimmune disorders, we started the appropriate screening for each disease. Therefore, most of the diagnoses of NEAD were established after RPL, but they were anyway confirmed by the appropriate specialist in our institution.

According to the ethical rules of "Sapienza" University of Rome and to the guidelines in the Declaration of Helsinki, this retrospective study required no intervention and has been carried out on written informed consent and data collected remained strictly confidential and anonymous.

\section{Methods}

Diagnosis of HT. The diagnosis of HT was based on and confirmed by the presence of at least two of the following three criteria: high concentration of TPO-Abs (at least two times over the higher concentration considered as normal), a characteristic ultrasonographic pattern, and/or hypothyroidism $(20,21)$. Hypothyroidism has been defined according to ATA guidelines published in 2017 (thyrotropin [TSH] upper reference limit of $4.0 \mathrm{mU} / \mathrm{L}$ ) (21). Serum TSH, free thyroxine (fT4), and serum anti-TPO-Abs levels were derived from medical records.

Diagnosis of NEAD. The diagnosis of each single NEAD concurrent with HT was based on the clinical, serological, and histological examination obtained by biopsy when appropriate $(22,23)$ and/or according to the criteria approved by specific Guidelines or even Consensus Conferences (22-28). A patient with additional autoimmune disorder has been included in the study only when the diagnosis of each chronic autoimmune disease was confirmed by specialists (i.e., gastroenterologists, rheumatologists, dermatologists) who verified the diagnosis.

\section{Statistical Analysis}

Median (interquartile range [IQR]) has been used to describe continuous variables. Univariate analysis was performed by using chi-square test or the Fisher's Exact test for dichotomous and categorical variables while continuous variables have been analyzed by the non-parametric MannWhitney $U$ test. The Kruskal-Wallis non-parametric test has been used to analyze the medians of more than two groups. A multiple Poisson regression analysis was performed to calculate ORs and 95\% confidence intervals (CIs) for potential confounders of the association between presence of NEAD and abortion rate. For this purpose, fT4, TSH, TPOAbs, and age at the first abortion were tested in the model. Incidence rate ratios and their CIs were calculated. All the calculations have been made by using the statistical package STATA 15.0 or the INSTAT GraphPad Prism 5.0 software for Windows.

\section{Results}

Overall, 516 out of 3480 women experienced at least one miscarriage and among them, 187 women were excluded for the presence of known conditions that might have caused the pregnancy loss such as fetal or parental chromosomal abnormalities, uterine anatomic abnormalities (congenital uterine anomalies, intrauterine adhesions, uterine fibroids, or polyps), ectopic pregnancies, diagnosed infections, heritable thrombophilia, and severe and chronic maternal diseases. We have also excluded patients who completed their pregnancies using low-molecular-weight heparin, aspirin, or corticosteroids. Within the remaining 329 patients with unexplained miscarriages, 87 patients met the criteria of RPL (5) and they represent the study group. More specifically, 65 of them had isolated HT and 22 women had NEAD concomitant with HT, diagnosed as described next (29). Their characteristics are shown in Table 1.

Women with isolated HT showed a rate of clinically evident RPL of $2.1 \%(n=65 / 3090)$; in contrast, in those women carrying both HT and NEAD, the prevalence of RPL was significantly higher $(n=22 / 390 ; 5.64 \%)(\mathrm{OR}=2.78$ [CI 1.70 4.57]; $p<0.0001$ ) (Fig. 1). No statistically significant dif- $<\mathrm{F} 1$ ferences were found when comparing the age at the time of the first miscarriage in patients with isolated HT and in those 
Table 1. Thyroid Parameters in Woman with Recurrent Pregnancy Loss Subdivided in Hashimoto's Thyroiditis IsOlated and Concurrent with Non-endocrine Autoimmune Disorders at the Time of The First Miscarriage

\begin{tabular}{lccc}
\hline & Isolated $H T$ & $H T+N E A D$ & $\mathrm{p}$ \\
\hline TSH (mU/L) & $2.65(1.05-5.62)$ & $1.87(1.28-5.03)$ & 0.94 \\
fT4 (ng/dL) & $1.20(1.02-1.40)$ & $1.00(0.88-1.09)$ & 0.24 \\
TPO-Abs & $350(147-996)$ & $520(133-1000)$ & 0.92 \\
\hline
\end{tabular}

Data are expressed as median values (interquartile range).

HT, Hashimoto's thyroiditis; fT4, free thyroxine; NEAD, nonendocrine autoimmune disorders; TPO-Abs, thyroid peroxidase antibodies; TSH, thyrotropin.

bearing HT+NEAD (median age $=28$ and 31 years, respectively; $p=0.19$ ).

As hypothyroidism itself may represent a cause of RPL (30), we examined the thyroid function of these women at the time of the first miscarriage. Overall, 57 out of 87 patients $(66 \%)$ were euthyroid with a median TSH of $1.50 \mathrm{mU} / \mathrm{L}$ $(\mathrm{IQR}=0.62-2.40)$. This group consisted of women treated $(n=23)$ and untreated $(n=34)$ with T4. No difference in the distribution of T4-treated patients was observed between women with isolated HT (16/41) and women with HT+NEAD (7/16; $p=0.77)$. In euthyroid women, a higher prevalence of RPL has been observed in patients with HT and NEAD as compared with patients with isolated HT (16/390 vs. $41 / 3090 \mathrm{OR}=3.181$ [CI 1.77-5.72]; $p<0.0001)$

F2 (Fig. 2A). Conversely, in hypothyroid women $(n=30)$ with a median TSH of $6.18 \mathrm{mU} / \mathrm{L}(\mathrm{IQR}=5.61-11.60)$, the rate of RPL was similar in patients with HT isolated or concurrent with NEAD (24/3090 vs. 6/390; $p=0.2140)$ (Fig. 2B). These findings would not have been changed if the hypothyroid women with RPL had been further subdivided into subclinical $(n=21 ; p=0.17)$ and overt hypothyroidism $(n=9$; $p=0.85)$.

To evaluate the correlation between the anti-TPO-Abs titer and the number of miscarriages, our study population has been stratified into four subgroups: (1) patients with isolated HT and two RPL events (46/65); (2) patients with isolated HT and three or more RPL events (19/65); (3) patients with

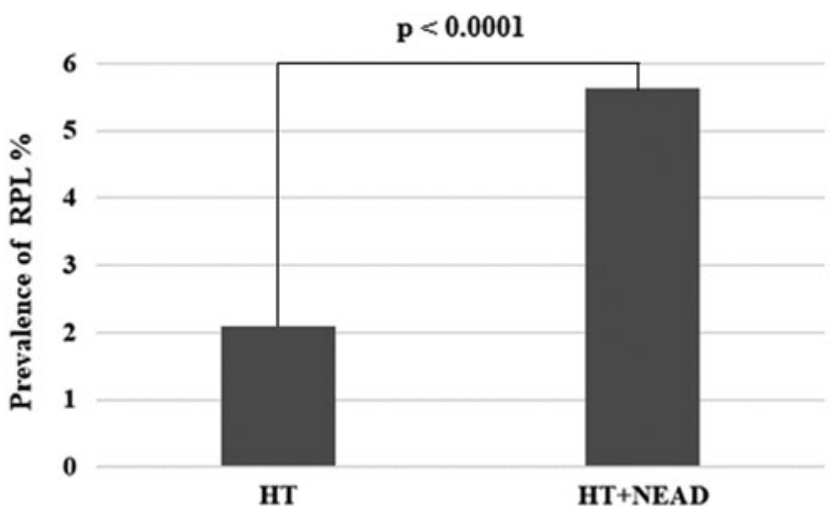

FIG. 1. Prevalence of RPL in patients with isolated HT and HT accompanied with NEAD. HT, Hashimoto's thyroiditis; NEAD, non-endocrine autoimmune disorders; RPL, recurrent pregnancy loss.
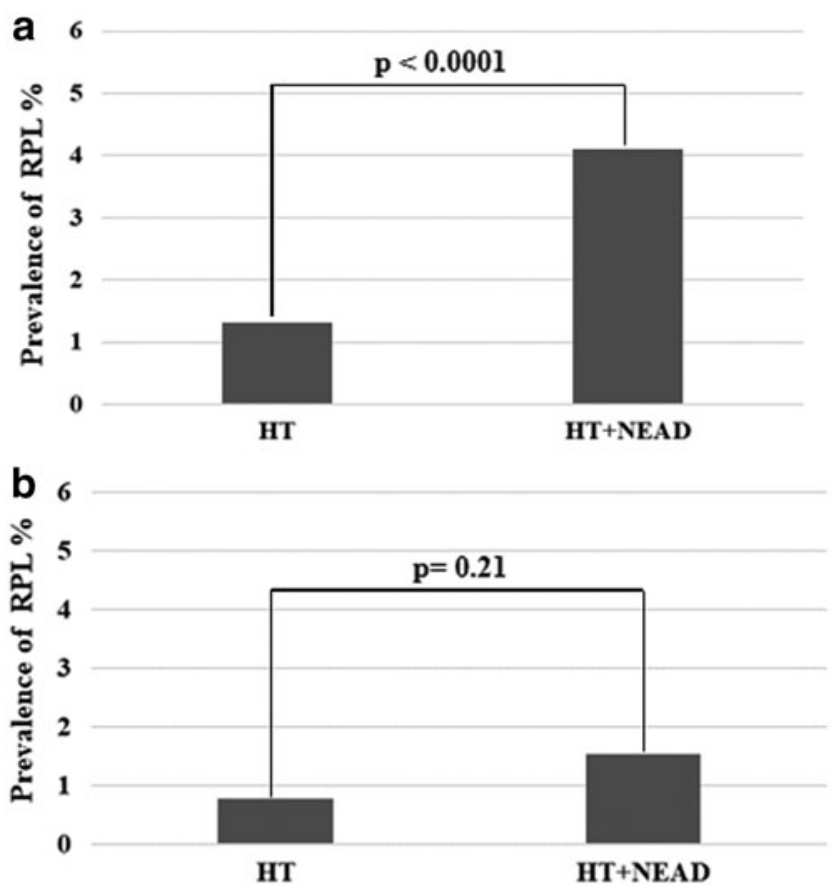

FIG. 2. Prevalence of RPL in euthyroid (A) and hypothyroid (B) patients with isolated HT and HT with concurrent NEAD.

HT+NEAD and two RPL events (16/22); and (4) patients with HT+NEAD and three or more RPL events $(6 / 22)$. No statistically significant differences have been observed $(p=0.99)$ when the TPO-Abs median titer was compared in these subgroups. Thus, the number of miscarriages was independent from the autoantibody titer both in women with isolated HT as well as in women with HT and NEAD.

We have considered the values of fT4, TSH, TPO-Abs, and age of the first abortion as possible confounders. No statistically significant confounders were identified through multiple Poisson regression analysis.

The APS, which may be concurrent in patients with HT (31), is, by itself, a cause of miscarriage, often due to the thrombosis of the placental vessels. We hypothesized that the presence of APS might justify the higher prevalence of miscarriage in our patients with poly-autoimmunity. In our sample, an APS has been diagnosed in 18 out of 390 women with $\mathrm{HT}+\mathrm{NEAD}$, but RPL occurred in only three of them. As depicted in Table 2 , the rate of RPL is similar in each NEAD, $४ \mathrm{~T} 2$ ranging from $5.5 \%$ to $8.3 \%$, with the expected exception of APS (13.6\%), because of its intrinsic pro-abortive action. Despite that, in patients with HT+NEAD, APS was present in a small fraction of women with RPL (3/22) while the remaining women with RPL were affected by different concurrent autoimmune disorders (Table 2). In fact, even subtracting the three patients with APS, a significantly higher rate of RPL was observed in patients with poly-autoimmunity than in patients with isolated HT $(p=0.0013)$.

\section{Discussion}

The findings of this study show an increased rate of recurrent miscarriage in patients with poly-autoimmune disorders as compared with patients affected by isolated HT. 
Table 2. Non-endocrine Autoimmune Disorders CONCURRENT WITH Hashimoto's ThyroIDITIS in Study Group Patients with RecurRent PREgNANCY LOSS

\begin{tabular}{|c|c|c|c|}
\hline & $\begin{array}{c}\text { NEAD } \\
\text { with } \\
\text { RPL }\end{array}$ & $\begin{array}{l}\text { Total } \\
\text { NEAD }\end{array}$ & \\
\hline & $\mathrm{n}=22$ & $\mathrm{n}=390$ & $\%^{\mathrm{a}}$ \\
\hline \multicolumn{4}{|l|}{ Hashimoto's thyroiditis } \\
\hline Chronic autoimmune gastritis & 10 & 124 & 8.1 \\
\hline Antiphospholipid syndrome & 3 & 22 & 13.6 \\
\hline Coeliac disease & 3 & 55 & 5.5 \\
\hline Sjögren's syndrome & 2 & 26 & 7.7 \\
\hline Rheumatoid arthritis & 2 & 27 & 7.4 \\
\hline $\begin{array}{l}\text { Undifferentiated connective tissue } \\
\text { disease }\end{array}$ & 1 & 12 & 8.3 \\
\hline Myasthenia gravis & 1 & 1 & $\mathrm{n} / \mathrm{a}^{\mathrm{b}}$ \\
\hline
\end{tabular}

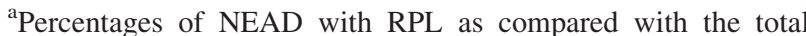
NEAD population. The list of remaining autoimmune disorders concurrent with HT is wise, but it is mostly represented by nonsegmental vitiligo and multiple sclerosis. Patients bearing these associated disorders had no RPL.

${ }^{\mathrm{b}}$ No other patients had myasthenia gravis in the whole sample and therefore that percentage is not applicable.

RPL, recurrent pregnancy loss.

The contribution of additional autoimmune disorders to RPL in women with HT has been scarcely assessed and, up to now, by detecting the co-presence of non-organ specific autoantibodies, since about $20 \%$ of women with thyroid autoimmunity had one or more of these antibodies (32). However, whether RPL in women with HT is directly linked to the presence of these non-organ specific autoantibodies or to the presence of a definite autoimmune disease is still unclear (32). In the study presented here, the RPL occurrence has been analyzed in HT patients in whom a definite diagnosis of NEAD has been established (i.e., not only with autoantibodies positivity). The association of HT and NEAD has been initially described by Neufeld and Blizzard and more recently reappraised by Boelaert et al. (33). Among these, the most common are atrophic gastritis/pernicious anemia (34), rheumatoid arthritis (35), vitiligo (36), celiac disease (37),

AU5 and LES/APS $(31,38)$.

In the study presented here, we observed that hypothyroidism represents a paramount factor in determining RPL, as already known $(30,39)$. In fact, on subdivision of the whole sample based on thyroid function, the different rate of RPL between HT patients and those with HT+NEAD is still evident in euthyroid women but disappears in hypothyroid patients. This is in keeping with the described effect of hypothyroidism (30) on pregnancy outcomes that, in this setting, may prevail over the immunological issues. On the contrary, immunological derangement may prevail in euthyroid women, determining a rate of RPL in women with HT and NEAD that is higher than in women with isolated HT (39).

Although a role of thyroid autoantibodies in determining RPL had been repeatedly claimed $(6,14,31,40)$, we did not observe significant correlations between the levels of antiTPO-Abs and the number of miscarriage events. Similarly, there were no differences in the level of these autoantibodies observed in women with isolated HT or with concurrent
NEAD in relation with the presence of RPL. These findings mirror the seminal observations of Stagnaro-Green et al. (41), who found no direct relationship between autoantibody levels and pregnancy outcomes for either anti-thyroglobulin or antiTPO-Abs. In this view, anti-TPO-Abs positivity (14) may only be an indicator of a complex disruption of self-tolerance, leading to pro-inflammatory responses and resulting in RPL $(17,18,39,40)$.

APS is a known cause of recurrent miscarriage $(6,31,40)$ through three main pathological mechanisms: the imbalance of the immune system with self-aggressive immune drift of the fetus, the direct disruption of trophoblasts, and/or placental thrombosis (40). Our data suggest that this syndrome may only partially explain the abortive events recognized in poly-autoimmune patients $(6,31)$. Most of the miscarriages were linked to apparently unrelated autoimmune disorders such as atrophic gastritis, connective tissue diseases, and celiac disease. It is noteworthy that the frequency of RPL in each NEAD closely reflects the distribution of these diseases in our 390 poly-autoimmune patients. This distribution is similar to the one described by Fallahi et al. (42). Therefore, the increased risk of recurrent miscarriages in poly-autoimmunity is likely due to a complex dysregulation of the cellular immunity that deserves further investigations $(9,43,17,18,32)$. The link between autoimmune thyroiditis and RPL has been suggested by means of different mechanisms but is, as yet, poorly understood $(3,43)$. In the pathogenesis of HT, an early polarization of proinflammatory Th17 lymphocytes seems to shift toward a Th1 polarization with proapoptotic activity (44). This activation requires an impaired response by both $\mathrm{B}$ and $\mathrm{T}$ regulatory cells $(45,46)$. As in autoimmune thyroid disease, a prevalent Th1 polarization has been described in women with RPL $(17,18)$. In women with HT, a significant increase in the endometrial $\mathrm{T}$ cell was also observed while the relative concentrations of both IL-4 and IL-10 in that tissue were decreased (43). The presence of a further autoimmune disorder may change the immunological features of isolated HT $(29,45)$. For example, in a previous study, an increased number of Breg cells, characterized by a reduced suppressive function, was associated with the simultaneous occurrence of NEAD with HT, independently from the concurrent disease (46). However, this mechanism is far from being clarified and requires further characterization of the described events.

We acknowledge that our study has some limitations. First, the retrospective nature of this study does not exclude the presence of some diagnostic bias. However, we have excluded autoantibody positivity as a unique diagnostic criterion and included only patients with histologic or consensus-based diagnoses of an autoimmune disorder, confirmed by an expert specialist. So far, we have restricted any possible misclassification of each autoimmune disorder included in the NEAD. The use of these restrictive criteria reduced the number of a study group encompassing patients with a rare association of autoimmune disorders with RPL even more.

In conclusion, we have observed that in women with HT, the concurrent presence of further NEAD is characterized by a higher risk of RPL. The presence of an anti-phospholipid syndrome may explain some, but not all, events of repeated pregnancy loss in these patients. Finally, the occurrence of an unexplained miscarriage in women with autoimmune thyroiditis should drive the search for additional autoimmune disorders. 


\section{Acknowledgment}

The authors are deeply indebted to Professor Corrado De Vito for his invaluable assistance in the statistical analysis of the data.

\section{Author Disclosure Statement}

M.C. and C.V. received travel expenses and honorarium to attend international Meetings and Boards from IBSA, 6915 Pambio Noranco, $\mathrm{CH}$. The remaining authors have nothing to disclose.

\section{Funding Information}

This study has been supported by Sapienza University of Rome, Italy (Progetti di Ateneo 2017 n. RM11715C71A02A85).

\section{References}

1. Griebel CP, Halvorsen J 2005 Management of spontaneous abortion. Am Fam Physician 72:1243-1250.

2. Larsen EC, Christiansen OB, Kolte AM, Macklon N 2013 New insights into mechanisms behind miscarriage. BMC Med 11:154.

3. Stagnaro-Green A, Glinoer D 2004 Thyroid autoimmunity and the risk of miscarriage. Best Pract Res Clin Endocrinol Metab 18:167-181.

4. Pandey MK, Rani R 2005 An update in recurrent spontaneous abortion. Arch Gynecol Obstet 272:95-108.

5. The ESHRE Guideline Group on RPL, Atik RB, Christiansen OB, Elson J, Kolte AM, Lewis S, Middeldorp S, Nelen W, Peramo B, Quenby S, Vermeulen N, Goddijn M 2018 ESHRE guideline: recurrent pregnancy loss. Human Reprod Open 2018:1-12.

6. De Carolis C, Greco E, Guarino MD, Perricone C, Dal Lago A, Giacomelli R, Fontana L, Perricone R 2004 Antithyroid antibodies and antiphospholipid syndrome: evidence of reduced fecundity and of poor pregnancy outcome in recurrent spontaneous abortion. Am J Reprod Immunol 52:263-266.

7. Grimstad F, Krieg S 2016 Immunogenetic contributions to recurrent pregnancy loss. J Assist Reprod Genet 33:833847.

8. Caturegli P, De Remigis A, Rose NR 2014 Hashimoto thyroiditis: clinical and diagnostic criteria. Autoimmun Rev 13:391-397.

9. Weetman AP 2010 Immunity, thyroid function and pregnancy: molecular mechanisms. Nat Rev Endocrinol 6:311318.

10. Weetman AP 2004 Autoimmune thyroid disease. Autoimmunity 37:337-340.

11. Arachchillage DR, Laffan M 2017 Pathogenesis and management of antiphospholipid syndrome. Br J Haematol 178: 181-195.

12. Lazarus JH, Premawardhana LD 2005 Screening for thyroid disease in pregnancy. J Clin Pathol 58:449-452.

13. Poppe K, Velkeniers B, Glinoer D 2007 Thyroid disease and female reproduction. Clin Endocrinol (Oxf) 66:309-321.

14. Poppe K, Velkeniers B, Glinoer D 2008 The role of thyroid autoimmunity in fertility and pregnancy. Nat Clin Pract Endocrinol Metab 4:394-405.

15. Prummel MF, Wiersinga WM 2004 Thyroid autoimmunity and miscarriage. Eur J Endocrinol 150:751-755.
16. Toth B, Jeschke U, Rogenhofer N, Scholz C, Würfel W, Thaler CJ, Makrigiannakis A 2010 Recurrent miscarriage: current concepts in diagnosis and treatment. J Reprod Immunol 85:25-32.

17. Makhseed M, Raghupathy R, Azizieh F, Omu A, AlShamali E, Ashkanani L 2001 TH1 and TH2 cytochine profiles in recurrent aborters with successfull pregnancy and with subsequent abortion. Hum Reprod 16:2219-2226.

18. Beaman KD, Ntrivalas E, Mallers TM, Jaiswal MK, KwakKim J, Gilman-Sachs A 2012 Immune etiology of recurrent pregnancy loss and its diagnosis. Am J Reprod Immunol 67:319-325.

19. Betterle C, Zanchetta R 2003 Update on autoimmune polyendocrine syndromes (APS). Acta Biomed 74:9-33.

20. Pearce EN, Farwell AP, Braverman LE 2003 Thyroiditis. N Engl J Med 348:2646-2655.

21. Alexander EK, Pearce EN, Brent GA, Brown RS, Chen H, Dosiou C, Grobman WA, Laurberg P, Lazarus JH, Mandel SJ, Peeters RP, Sullivan S 20172017 Guidelines of the American Thyroid Association for the diagnosis and management of thyroid disease during pregnancy and the postpartum. Thyroid 27:315-389.

22. Neumann WL, Coss E, Rugge M, Genta R 2013 Autoimmune atrophic gastritis- pathogenesis, pathology and management. Nat Rev Gastroenterol Hepatol 10:529-541.

23. Mueller RB, Schiff M, Kaegi T, Finckh A, Haile SR, Schulze-Koops H, von Kempis J; Swiss Clinical Quality Management (SCQM) physicians 2015 The new 2010 ACR/EULAR criteria as predictor of clinical and radiographic response in patients with early arthritis. Clin Reumatol 34:51-59.

24. Sumida T, Azuma N, Moriyama M, Takahashi H, Asashima $\mathrm{H}$, Honda $\mathrm{F}$, Abe $\mathrm{S}$, Ono $\mathrm{Y}$, Hirota $\mathrm{T}$, Hirata $\mathrm{S}$, Tanaka Y, Shimizu T, Nakamura H, Kawakami A, Sano H, Ogawa Y, Tsubota K, Ryo K, Saito I, Tanaka A, Nakamura S, Takamura E, Tanaka M, Suzuki K, Takeuchi T, Yamakawa N, Mimori T, Ohta A, Nishiyama S, Yoshihara T, Suzuki Y, Kawano M, Tomiita M, Tsuboi H 2018 Clinical practice guideline for Sjögren's syndrome 2017. Mod Rheumatol 28:383-408.

25. Mosca M, Tani C, Vagnani S, Carli L, Bombardieri S 2014 The diagnosis and classification of undifferentiated connective tissues diseases. J Autoimmun 48:50-52.

26. Sussman J, Farrugia ME, Maddison P, Hill M, Leite MI, Hilton-Jones D 2018 The Association of British Neurologists' myasthenia gravis guidelines. Ann N Y Acad Sci 1412: $166-169$.

27. Bai JC, Fried M, Corazza GR, Schuppan D, Farthing M, Catassi C, Greco L, Cohen H, Ciacci C, Eliakim R, Fasano A, González A, Krabshuis JH, LeMair A 2013 World Gastroenterology Organisation global guidelines on celiac disease. J Clin Gastroenterol 47:121-126.

28. Miyakis S, Lockshin MD, Atsumi T, Branch DW, Brey RL, Cervera R, Derksen RH, DE Groot PG, Koike T, Meroni PL, Reber G, Shoenfeld Y, Tincani A, Vlachoyiannopoulos PG, Krilis SA 2006 International consensus statement on an update of the classification criteria for definite antiphospholipid syndrome (APS). J Thromb Haemost 4:295306.

29. Santaguida MG, Nardo S, Del Duca SC, Lococo E, Virili C, Gargano L, Lenti L, Centanni M 2011 Increased interleukin-4-positive lymphocytes in patients with Hashimoto's thyroiditis and concurrent non-endocrine autoimmune disorders. Clin Exp Immunol 165:148-154. 
30. Negro R, Schwartz A, Gismondi R, Tinelli A, Mangieri T, Stagnaro-Green A 2010 Increased pregnancy loss rate in thyroid antibody negative women with TSH levels between 2.5 and 5.0 in the first trimester of pregnancy. J Clin Endocrinol Metab 95:E44-E48.

31. Versini M 2017 Thyroid autoimmunity and antiphospholipid syndrome: not such a trivial association. Front Endocrinol (Lausanne) 8:175.

32. Pratt D, Novotny M, Kaberlein G, Dudkiewicz A, Gleicher N 1993 Antithyroid antibodies and the association with non-organ-specific antibodies in recurrent pregnancy loss. Am J Obstet Gynecol 168:837-841.

33. Boelaert K, Newby PR, Simmonds MJ, Holder RL, CarrSmith JD, Heward JM, Manji N, Allahabadia A, Armitage M, Chatterjee KV, Lazarus JH, Pearce SH, Vaidya B, Gough SC, Franklyn JA 2010 Prevalence and relative risk of other autoimmune diseases in subjects with autoimmune thyroid disease. Am J Med 123:183.e1-183.e9.

34. Cellini M, Santaguida MG, Virili C, Capriello S, Brusca N, Gargano L, Centanni M 2017 Hashimoto's thyroiditis and autoimmune gastritis. Front Endocrinol (Lausanne) 8: 92.

35. Feldt-Rasmussen U 2015 Increased risk of thyroid autoimmunity in rheumatoid arthritis. Endocrine 50:4-5.

36. Ferrari SM, Fallahi P, Santaguida G, Virili C, Ruffilli I, Ragusa F, Centanni M, Antonelli A 2017 Circulating CXCL10 is increased in non-segmental vitiligo, in presence or absence of autoimmune thyroiditis. Autoimmun Rev 16: 946-950.

37. Ferrari SM, Elia G, Virili C, Centanni M, Antonelli A, Fallahi P 2017 Systemic lupus erythematosus and thyroid autoimmunity. Front Endocrinol (Lausanne) 8:138.

38. Virili C, Bassotti G, Santaguida MG, Iuorio R, Del Duca SC, Mercuri V, Picarelli A, Gargiulo P, Gargano L, Centanni M 2012 Atypical celiac disease as cause of increased need for thyroxine: a systematic study. J Clin Endocrinol Metab 97:E419-E422.

39. De Leo S, Pearce EN 2018 Autoimmune thyroid disease during pregnancy. Lancet Diabetes Endocrinol 6:575-586.
40. Chen L, Hu R 2011 Thyroid autoimmunity and miscarriage: a meta-analysis. Clin Endocrinol (Oxf) 74:513-519.

41. Stagnaro-Green A, Roman SH, Cobin R, Hel-Harazy E, Alvarez-Marfany M, Davies TF 1990 Detection of at-risk pregnancy by means of highly sensitive assays for thyroid autoantibodies. JAMA 264:1422-1425.

42. Fallahi P, Ferrari SM, Ruffilli I, Elia G, Biricotti M, Vita R, Benvenga S, Antonelli A 2016 The association of other autoimmune diseases in patients with autoimmune thyroiditis: review of the literature and report of a large series of patients. Autoimmun Rev 15:1125-1128.

43. Twig, Shina A, Amital H, Shoenfeld Y 2012 Pathogenesis of infertility and recurrent pregnancy loss in thyroid autoimmunity. J Autoimmun 38:J275-J281.

44. Figueroa-Vega N, Alfonso-Pérez M, Benedicto I, SánchezMadrid F, González-Amaro R, Marazuela M 2010 Increased circulating pro-inflammatory cytokines and Th17 lymphocytes in Hashimoto's thyroiditis. J Clin Endocrinol Metab 95:953-962.

45. Li D, Cai W, Gu R, Zhang Y, Zhang H, Tang K, Xu P, Katirai F, Shi W, Wang L, Huang T, Huang B 2013 Th17 cell plays a role in the pathogenesis of Hashimoto's thyroiditis in patients. Clin Immunol 149:411-420.

46. Santaguida MG, Gatto I, Mangino G, Virili C, Stramazzo I, Fallahi P, Antonelli A, Segni M, Romeo G, Centanni M 2017 BREG cells in Hashimoto's thyroiditis isolated or associated to further organ-specific autoimmune diseases. Clin Immunol 184:42-47.

Address correspondence to: Marco Centanni, MD

Department of Medico-Surgical Sciences and Biotechnologies

"Sapienza" University of Rome

Corso della Repubblica 79

Latina 04100

Italy

E-mail: marco.centanni@uniroma1.it 


\section{AUTHOR QUERY FOR THY-2019-0456-VER9-CELLINI_1P}

AU1: Please identify (highlight or circle) all authors' surnames for accurate indexing citations.

AU2: Please confirm the correctness of authors' affiliations.

AU3: The Publisher requests for readability that no paragraph exceeds 15 typeset lines. Please check for long paragraphs and divide where needed.

AU4: Refs. 11-43 have been renumbered for sequential order of citations in the text and list. Please check.

AU5: Please expand "LES." 